\title{
An Approach for Uncertainty Quantification and Management of Unmanned Aerial Vehicle Health
}

\author{
Matteo Corbetta ${ }^{1}$ and Chetan S. Kulkarni ${ }^{2}$ \\ ${ }^{1,2}$ SGT Inc., NASA Ames Research Center, Moffett Field, 94035 CA, United States \\ matteo.corbetta@nasa.gov \\ chetan.s.kulkarni@nasa.gov
}

\begin{abstract}
The increasing interest in low-altitude unmanned aerial vehicle (UAV) operations is bringing along safety concerns. Performance of small, low-cost UAVs drastically changes with type, size and controller of the vehicle. Their reliability is significantly lower when compared to reliability of commercial aircraft, and the availability of on-board sensors for health and state awareness is extremely limited due to their size and propulsion capabilities. Uncertainty plays a dominant role in such a scenario, where a variety of UAVs of different size, propulsion systems, dynamic performance and reliability enters the low-altitude airspace. Unexpected failures could have dangerous consequences for both equipment and humans within that same airspace. As a result, a number of research tasks and methodologies are being proposed in the area of UAV dynamic modeling, health and safety monitoring, but uncertainty quantification is rarely addressed. Thus, this paper proposes a perspective towards uncertainty quantification for autonomous systems, giving special emphasis to UAV health monitoring application. A formal approach to classify uncertainty is presented; it is utilized to identify the uncertainty sources in UAVs health and operations, and then map uncertainty within a predictive process. To show the application of the methodology proposed here, the design of a model-based powertrain health monitoring algorithm for small-size UAVs is presented as case study. The example illustrates how the uncertainty quantification approach can help the modeling strategy, as well as the assessment of diagnostic and prognostic performance.
\end{abstract}

\section{INTRODUCTION}

The number of unmanned aerial vehicles (UAVs) entering the low-altitude airspace is expected to increase in the next decade (Kopardekar et al., 2016; FAA, 2018). This forecast, driven by current interests in autonomous UAV operations

\footnotetext{
Matteo Corbetta et al. This is an open-access article distributed under the terms of the Creative Commons Attribution 3.0 United States License, which permits unrestricted use, distribution, and reproduction in any medium, provided the original author and source are credited.
}

like package delivery, surveillance, etc., as well as future urban air mobility, suggests the need of systematic approaches to enable autonomous UAV operations efficiently and safely. Such a need is motivated by multiple factors. As addressed in (Kopardekar et al., 2016), unmanned systems will enter areas originally used by traditional, manned aviation. However, infrastructure and integration requirements were not developed to accommodate a mix of different vehicles and systems. Small, low-cost UAVs, which are likely to be utilized for package delivery and other operations, do not guarantee high reliability standards (King, Bertapelle, \& Moses, 2005; Freeman \& Balas, 2014; Johry \& Kapoor, 2016), suggesting that high failure rates may be expected, especially when compared to commercial aviation. Focusing on urban air mobility, PHM tools are paramount to ensure maximum human safety on ground as well as on-board and minimize failure rates and service disruption.

The effect of this growing interest in low-altitude operations is a number of research activities on autonomous UAVs, including new design, reliability, efficiency, and autonomous functions. Examples of such works can be found in (Hoffmann, Huang, Waslander, \& Tomlin, 2007) for flight dynamics and control, (Langelaan, Alley, \& Neidhoefer, 2011; Glasheen, Pinto, Steiner, \& Frew, 2019) for wind field estimation, (Krishnakumar et al., 2017) on the safety of UAV operations, and (Balaban et al., 2017) for dynamic routing and decision making. More generally, interests in autonomous vehicles also generated a number of system-level research on the safety of the national airspace, as in (Liu \& Goebel, 2018). Some system health management and PHM concepts for UAVs were discussed in (Jing \& Haifeng, 2013; Walker, 2010), while an early study on fault detection for unmanned vehicles was presented in (Drozeski, Saha, \& Vachtsevanos, 2005).

Besides studies of uncertainty affecting aircraft routing (Jun \& D'Andrea, 2003) and methods for conflict avoidance (Albaker \& Rahim, 2009), methodologies to handle and approach uncertainty affecting UAV systems and operations has 
been rarely explored. The work by Sankararaman et. al in (Sankararaman, 2017) presented uncertainty sources influencing UAV operations were identified and examples of decision making strategy based on those uncertainty sources were presented.

This work proposes a methodology for uncertainty identification and quantification, with focus on PHM applications for autonomous unmanned vehicles. The paper presents a formal approach to uncertainty representation and quantification, where the main uncertainty sources are identified and then mapped into a predictive estimation process, where the input-output relationships of predictive estimation are discussed. This research leveraged material in (Smith, 2013), and previous works on uncertainty for PHM applications presented in (Sankararaman, 2015; Goebel, 2017). Besides the process has been developed in the context of autonomous UAVs, it can be easily translated and implemented to other systems. The predictive estimation process is applied to a physics-based powertrain health monitoring system composed of a Lithium-ion (Li-ion) battery, electronic speed controller (ESC), and brushless DC motor. The proposed approach helps mapping the input-output relationships of the powertrain health monitoring system and helps modeling of uncertainties in each of its elements. The uncertainty can then be propagated through the different model equations to quantify the uncertainty of the system dynamics in nominal conditions. The introduction of synthetic faults in the system will help quantitative assessments of the health monitoring system's performance in presence of uncertainty.

\section{FORMAL APPROACH TO UNCERTAINTY QUANTIFI- CATION}

This section discusses some key aspects of uncertainty quantification from a global perspective. Such a formal approach enables the identification, interpretation, and classification of different uncertainty sources affecting the predictive estimation process. Although both aleatory and epistemic uncertainty are considered, a source of uncertainty will be categorized as aleatory or epistemic only if beneficial for the discussion. This approach will serve as the foundation for the identification of uncertainty sources in the UAV powertrain health monitoring system discussed in Section 3.

A high-level subdivision of uncertainty sources is presented, combining information from (Smith, 2013), Chapter 1, and (Goebel, 2017). Besides in this holistic perspective, the sources should be then considered relative to the specificity of the application, since some of them may not be relevant for some scenarios.

The macro-categories model, method, measure, and input have been chosen to represent the uncertainty sources of predictive estimation. Each of them comprises of sub-categories which are depicted in figure 1, and they are discussed in the next subsections.

\subsection{Model}

Model uncertainty has been divided in model abstraction and model parameters. Model abstraction refers to the hypotheses introduced during model development with the intent of representing reality and physical processes through a set of equations. Those hypotheses include neglecting or simplifying (for example, through linearization, reduced order models) physical phenomena, environmental effects, and other external factors that may interact with the system, but their effect on the quantities of interest (QoIs) is believed to be limited.

Model parameters include fixed or variable coefficients required to estimate the output QoIs from the model. They may be unknown, or believed to fall within a range of values, or again defined by random variables and therefore represented through probability density functions (pdfs). They may depend on system's properties, and they may also evolve over time because of degradation phenomena.

\subsection{Methods}

Here, methods refers to the collection of algorithms and computation tools utilized within a certain model to compute the QoIs, to interpolate or extrapolate input variables from input data, or to perform prediction by propagating information through models or in time domain. The sources of uncertainty belonging to this category have been divided into algorithms and numerical errors.

Algorithms for estimation, interpolation or extrapolation in multi-dimensional spaces introduce uncertainty because they may converge to sub-optimal solutions trying to minimize errors or cost functions. Such cost functions may be nonconvex or complex hyper-surfaces if the system is nonlinear. As a result, different runs of the algorithms may generate different results because the algorithms remain trapped in local minima. Numerical errors include roundoff or discretization errors, which is typically small when compared to other sources (Smith, 2013), but it may not be negligible for certain engineering or physics applications.

\subsection{Measures}

This category includes measure incompleteness, uncertainty in measurement equipment, which typically translates into sensor resolution, accuracy and precision, and systematic errors generated by the measuring process, sensor installation and human error (if humans are involved in the measuring process). 


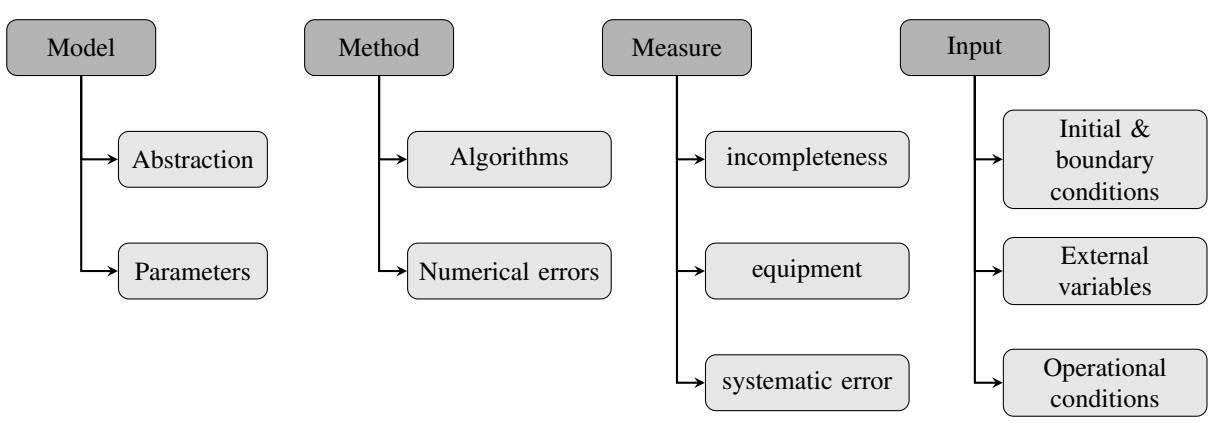

Figure 1. High-level classification of uncertainty sources encountered in a predictive process.

\subsection{Input}

The definition of input used in this work includes timedependent variables, initial and boundary conditions, and exogenous forces that may interact with the system and therefore affects its dynamics. Some inputs are actually operational requirements defined according to the system's intended function, and they are called operational input (e.g., desired trajectory of an autonomous vehicle or angular speed of a rotating machinery). External inputs are generated by external forces or events which depend on the operational environment of the system. They can also be called environmental or exogenous input, and are typically characterized by large uncertainty. Initial and boundary conditions refer to both external and system's variables. For example, the initial degradation state of a system's component may be uncertain. If such a component is already in a degraded state, its progressive degradation during operation will likely be faster than if the component were healthy.

\subsection{Mapping uncertainty sources in the predictive esti- mation process}

Figure 2 shows a diagram of the high-level predictive process structure proposed in this work. The sources of uncertainty discussed in figure 1 are tightly connected to this structure. From left to right, we introduce the measure space, which represents quantities that have to be measured. Those split into system's variables and external variables. The former are the QoIs directly linked with the system's state or some system's property, while external variables are the QoIs representing external or exogenous inputs.

The input space represents those variables not belonging to the system that is being monitored but affect the system's dynamics. The input space overlaps with the measured space, since external variables have to be, somehow, estimated or measured. As discussed in subsection 2.4, operational variables define the intended or desired behavior of the system (e.g., the flight plan for an autonomous aircraft). Operational variables do not typically require measurement processes, and they are then placed outside of the measured space.

Measured space and Input space variables feed the representation space, which comprises of system's models and input models. While the introduction of the system's model is self-explanatory, the reason for incorporating input models is twofold. As stated earlier, models of engineering systems are often described by differential equations or complex systems of equations, and input specifications may not be written in a form that is directly implementable into those equations. For example, a flight plan for autonomous UAVs composed of way points and cruise speed needs to be converted into a desired trajectory profile if the aircraft's model (i.e., the system's model) is a 6 degrees-of-freedom equation of motion. Input models play a relevant role also for external variables. Some external variables affecting the system's dynamics may be hidden (not directly measurable), and therefore a model to extract the latent variable(s) from observations is necessary. Often, external variables are non-deterministic and affected by large uncertainty, and a model to perform quantification and look-ahead forecast becomes a necessity. Another example is represented by external input information available on a coarse grid, which is not suitable for the resolution of the models that are being adopted, and therefore require interpolation and extrapolation methods.

The fourth block represents the computing space, where numerical tools and models are combined to provide an estimation of the current QoIs of the system and their future values. Intuitively, the estimated prediction at the end of the process is affected by the uncertainty sources of all previous three spaces. The connection between input models and prediction methods has been highlighted since it is clear that input models help improving the forecast of QoIs by providing a statistical quantification (and therefore the related uncertainty) of future values of external variables. 


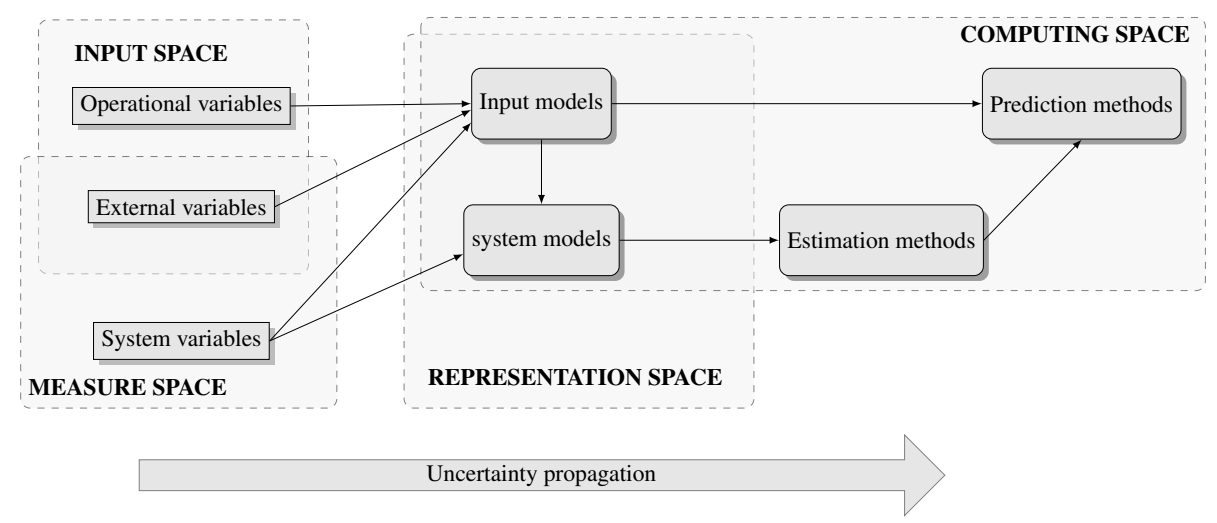

Figure 2. Predictive process structure. The bottom arrow describing uncertainty propagation does not indicate that uncertainty is introduced only in the measure and input spaces, but it rather indicates that all the elements in the predictive process introduce uncertainty, which increases from left to right.

\section{Application to an electrical powertrain HEALTH MONITORING SYSTEM}

In this section, the predictive process is applied to the design of a health monitoring system for electrical powertrain typically installed in small, low-cost UAVs. The health monitoring system composes of a electrochemistry model for Li-ion batteries developed in (Daigle \& Kulkarni, 2013), a model for the electronic speed controller (ESC) composed of a pulse-width modulation (PWM) system and 6 switches (Gorospe, Kulkarni, Hogge, Hsu, \& Ownby, 2017), and the dynamic model of the brushless DC motor utilized to actuate rotors. The diagram of the health monitoring system is presented in figure 3 .

\subsection{Overview of powertrain models}

This subsection summarizes key elements of each sub-model involved in the powertrain health monitoring system. For more information about the models and the equations they require, the reader is referred to Appendices A and B and references therein.

The ESC is modeled as ideal power inverter employing PWM and half-bridge drivers for each of the three phases within a control block. The PWM modulates three sine waves with carrier frequency $f$ and phase shifts $\varphi=\{0,2 \pi / 3,4 \pi / 3\}$ using a saw-tooth wave with frequency $f_{\text {st }}$. The output is a three phase voltage with duty cycle depending on $f$ and $f_{s t}$, (Pillay \& Krishnan, 1989; Holtz, 1992).

The model structure composes of two inputs; battery's output voltage $V$ and the three modulated square waves from PWM, $F_{1}, F_{2}$, and $F_{3}$. The switch matrix is a design property of the system, and therefore can be considered a model parameter. The three modulated square waves are pre-multiplied by the switch matrix and the battery's output voltage, as in Eq. (1). The output is a three-phase voltage $v_{a b}, v_{b c}, v_{c a}$, with phase shift of $\pm 2 / 3 \pi$ among one another, which becomes the input of the motor's electrical dynamic model (see the motor dynamic model in Appendix B).

$$
\left[\begin{array}{l}
v_{a b} \\
v_{b c} \\
v_{c a}
\end{array}\right]=V\left[\begin{array}{ccc}
1 & -1 & 0 \\
0 & 1 & -1 \\
-1 & 0 & 1
\end{array}\right]\left[\begin{array}{l}
F_{1} \\
F_{2} \\
F_{3}
\end{array}\right]
$$

Even if not specified, battery's output voltage $V$ and output PWM signals $F_{i}, \forall i=\{1,2,3\}$ are obviously time-varying.

The structures of the motor model and the battery discharge model are sets of first order differential equations, in the form:

$$
\dot{\boldsymbol{x}}=f_{\boldsymbol{p}}(\boldsymbol{x}, \boldsymbol{u})
$$

where $\boldsymbol{p}$ is the vector of model parameters and $\boldsymbol{u}$ is the input vector. State vectors are defined by $\boldsymbol{x}=$ $\left[q_{s, p}, q_{b, p}, q_{b, n}, q_{s, n}, V_{o}^{\prime}, V_{\eta, p}^{\prime}, V_{\eta, n}^{\prime}\right]^{T}$ and $\boldsymbol{x}=\left[i_{a}, i_{b}, \omega_{m}\right]^{T}$ for the battery and motor model, respectively. For the motor dynamic model, the vector of model parameters is $\boldsymbol{p}=\left[B, J, R_{s}, L_{M}, k_{e}\right]^{T}$ (each element is defined in Appendix B). For the battery model, the parameter vector is not reported for the sake of brevity (since uncertainty on those parameters is not discussed), and the reader is referred to (Daigle \& Kulkarni, 2013) for the complete set of parameters. The motor model parameters can be extracted from manufacturer data-sheets or estimated from experimental tests, while the parameters of the electro-chemistry battery model do require estimation from characterization test profiles. The input of the battery model is the applied current $\boldsymbol{u}=i$. The input of the motor model composes of: (i) two of the three-phase input voltages $\left(v_{a b}\right.$ and $v_{b c}$ ) (ii) three-phase back-emf voltages $e_{a}, e_{b}, e_{c}$, and (iii) load torque $T_{l}$, so $\boldsymbol{u}=\left[v_{a b}, v_{b c}, e_{a}, e_{b}, e_{c}, T_{l}\right]^{T}$. It should be noticed that motor model input vector is composed of both operational input, 


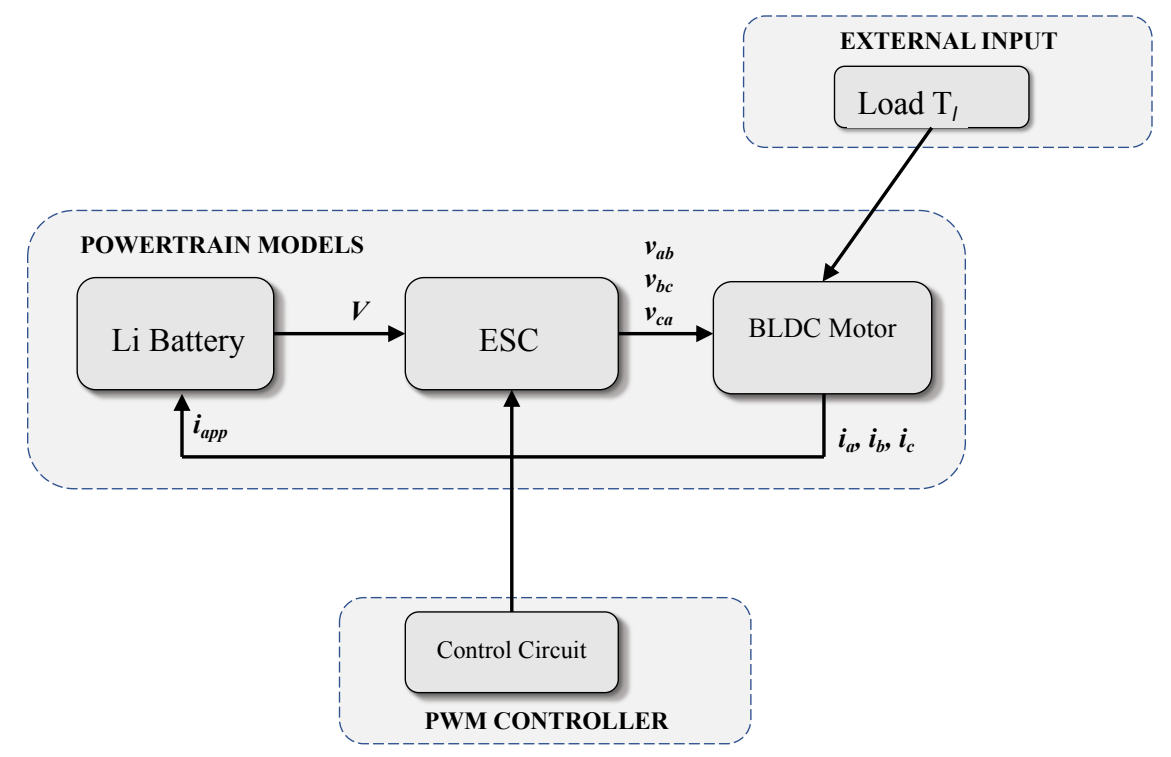

Figure 3. Framework for powertrain health monitoring uncertainty quantification.

that is the desired applied three-phase voltages, and external input $T l$ which depends on exogenous variables. Random variables representing the model error (or model noise) are discussed in more detail in Section 4.

\subsection{Simplifying assumptions}

For this specific application, un-modeled physical phenomena are neglected, therefore uncertainty referring to the model abstraction is not considered. Moreover, uncertainty in the battery model parameters is also neglected. The reason is the large number of model parameters; defining those as random variables without careful selection of the distribution functions or fine tuning of the dispersion indices (e.g., variance or parameter ranges) may easily lead to divergence of the model. Furthermore, probabilistic methods do not typically consider all model parameters as uncertain. Often, only a subset of them is reponsible for the majority of the output variance. A reasonable approach, left to future steps of this research, is to utilize local and global sensitivity analysis methods to indetify the effect of model output against parameter variations. Example of such approaches are available in (Sankararaman \& Mahadevan, 2013; Saltelli, Tarantola, Campolongo, \& Ratto, 2004).

This task is left to future refinement of the framework. Motor's model parameters can be described by random variables to encapsulate uncertainty on the model dynamics. Moreover, the motor model external input, $T l$, is likely to be affected by uncertainty, since external load may vary according to control commands and operating conditions. Additional details are discussed in Section 4.

For the sake of brevity, uncertainty in the observed quanti- ties due to measurement errors is not discussed, and is left to future works on the proposed framework. On the other hand, it should be noticed that a number of methodologies to incorporate measurement uncertainty are easily available in literature, such as a number of regression techniques that incorporate measurement errors (Smith, 2013), Bayesian filters (Arulampalam, Maskell, Gordon, \& Clapp, 2002), or datadriven methods such as Gaussian processes (Rasmussen \& Williams, 2006).

\section{REPRESENTING UNCERTAINTY IN THE POWER- TRAIN MODEL}

In this section, the approach to model the uncertainty in the powertrain health monitoring system is discussed. The models referred in this section are available in the Appendices and references therein.

\subsection{Battery}

The QoI is the output voltage $V$, which defines the energy introduced in the powertrain to produce the torque on the rotor and so the thrust to operate the vehicle. Given the complexity of the electro-chemistry battery model utilized in this work, a sampling-based approach is suggested. The state vector $\boldsymbol{x}$ from Eq. (A.9) is considered a random vector, where the Liions $q_{s, p}, q_{b, p}$ are defined by random variables. They represent the number of Li-ions on the positive side of the surface $q_{s, p}$ and bulk $q_{b, p}$ of the cell, respectively. Since $q_{s, n}, q_{b, n}$, as well as the voltages $V^{\prime}$, are derived quantities, they also become random variables. Independent, Gaussian pdfs have been utilized to compute random realizations of $q_{s, p}, q_{b, p}$ in a Monte Carlo fashion, using Euler's forward method: 


$$
\begin{aligned}
& q_{s, p, k}=q_{s, p, k-1}+\dot{q}_{s, p, k-1} \Delta t_{k-1}+\sigma_{q_{s, p}} \sqrt{\Delta t_{k-1}} r_{1} \\
& q_{b, p, k}=q_{b, p, k-1}+\dot{q}_{b, p, k-1} \Delta t_{k-1}+\sigma_{q_{b, p}} \sqrt{\Delta t_{k-1}} r_{2}
\end{aligned}
$$

where $r_{1}$ and $r_{2}$ are two independent realizations from the standard Normal distribution, and $k$ indicates the time step. Rates of change $\dot{q}_{s, p, k-1}$ and $\dot{q}_{b, p, k-1}$ are computed from Eq. (A.1). The random shocks introduced by $\sigma_{q_{l, p}} r_{1}$ and $\sigma_{q_{l, p}} r_{2}$ are scaled by $\sqrt{\Delta t}$ for consistency with Wiener process and Brownian motion used in stochastic differential equations (Lawler, 2010). By doing so, the variances of the two stochastic processes scale linearly with time. The two standard deviations $\sigma_{q_{s, p}}$ and $\sigma_{q_{b, p}}$ should be properly quantified to reflect the variability observed in experimental tests.

Figure 4 shows, as an example, the output of a single cell modeled using Eq. (2), with initial voltage $V_{0}=V(t=0) \sim$ $\mathcal{N}(4.6,0.316), \sigma_{q_{s, p}}^{2}=\sigma_{q_{b, p}}^{2}=10.0$, and assuming a constant discharge rate with required power $P=8 \mathrm{~W}$. The simulation parameters were $\Delta t=1 \mathrm{e}-1 \mathrm{~s}, N=1000$ samples, and final simulation time $100 \mathrm{~s}$. The kernel density estimate of the voltage at time $100 \mathrm{~s}$, computed with the Monte Carlo samples and Gaussian kernel with bandwidth equal to 0.125 , is compared against a Gaussian distribution, figure $4 \mathrm{~b}$.

\subsection{Electronic speed controller}

Uncertainty affecting the elements of the ESC in Eq. (1) is modeled in different ways. The sine wave carrier frequency $f$ could be represented by a deterministic value or a random variable with, typically, small uncertainty on its nominal value. It may decrease during operation because of slower switching actions of degraded MOSFETs (Gorospe et al., 2017), which are not modeled in the framework proposed here. Its degradation should be represented by a alwaysnegative rate of change, to ensure that $f$ is actually decreasing, and not increasing, over time. Consequently, uncertainty on the rate of change should reflect this monotonic behavior. A possible solution could include a negative log-Normallydistributed rate of change:

$$
f_{k}=f_{k-1}-\left.\frac{\mathrm{d} f}{\mathrm{~d} t}\right|_{k-1} e^{\eta},
$$

where the deterministic value $\mathrm{d} f / \mathrm{d} t$ is multiplied by $e^{\eta}$, and $\eta \sim \mathcal{N}\left(-\sigma_{\eta}^{2} / 2, \sigma_{\eta}^{2}\right)$ to ensure that $f$ is decreasing as time passes by. The minus sign - has been used to stress the fact that $f$ is decreasing, although it could be embedded in $\mathrm{d} f / \mathrm{d} t$. A suitable function to define the rate of change of $f$ should be tuned based on historical data on MOSFETs degradation. It should be noticed that this degradation is expected to be slow. For the specific application of UAV health monitoring,

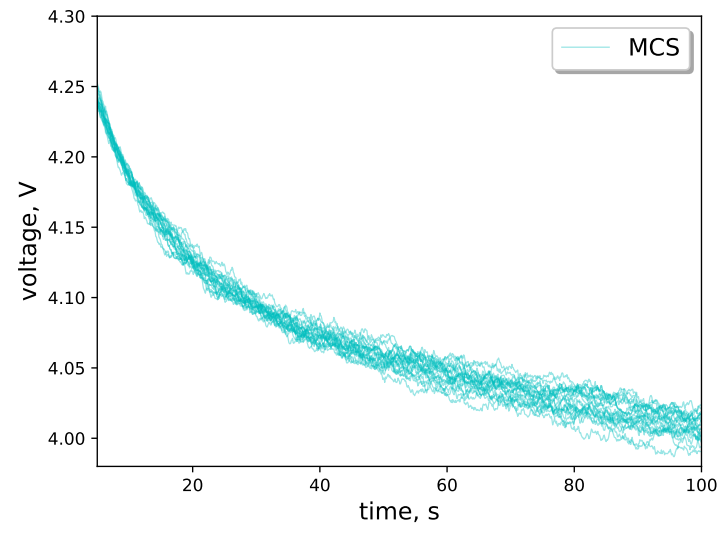

(a)

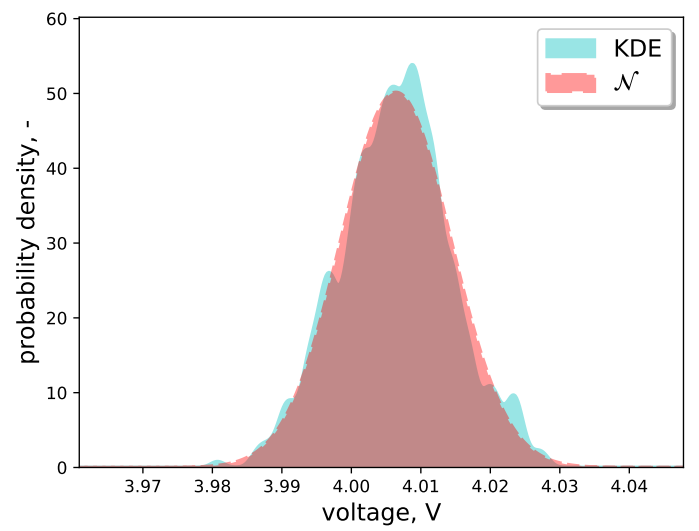

(b)

Figure 4. Simulation of battery discharge at constant power. Monte Carlo samples are shown in $4 \mathrm{a}$, while the resulting kernel density estimate is compared against a Gaussian distribution in $4 \mathrm{~b}$. Only a subset of all samples has been represented in $4 \mathrm{a}$ to appreciate the different paths.

changes in the PWM excitation frequencies are likely to be negligible in a single UAV flight, although the value of $f$ may be different from the nominal value due to previous aging.

An example of ESC signal computed with nominal carrier frequency $f=1 \mathrm{~Hz}$ and $f_{s t}=20 \mathrm{~Hz}$ is shown in figure 5 , where it is compared against the same signal with a reduced carrier frequency $f^{*}=0.95 f$. The low frequency values were chosen to appreciate the difference between the two cases.

We modeled switch failures by abrupt changes in the switch matrix in Eq. (1), using a typical reliability-based approach (Ginart, Brown, Kalgren, \& Roemer, 2009), where switch reliability is defined by failure rates $\lambda(t)$. The elements equal to 1 and -1 in the switch matrix will become 0 s when a failure of the corresponding MOSET switches occurs (Celaya, Saxena, Kulkarni, Saha, \& Goebel, 2012). By so doing, 


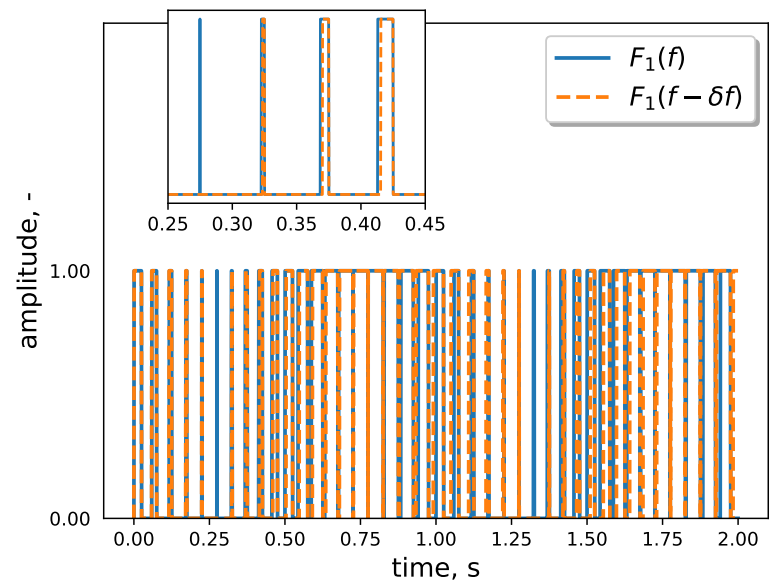

(a)
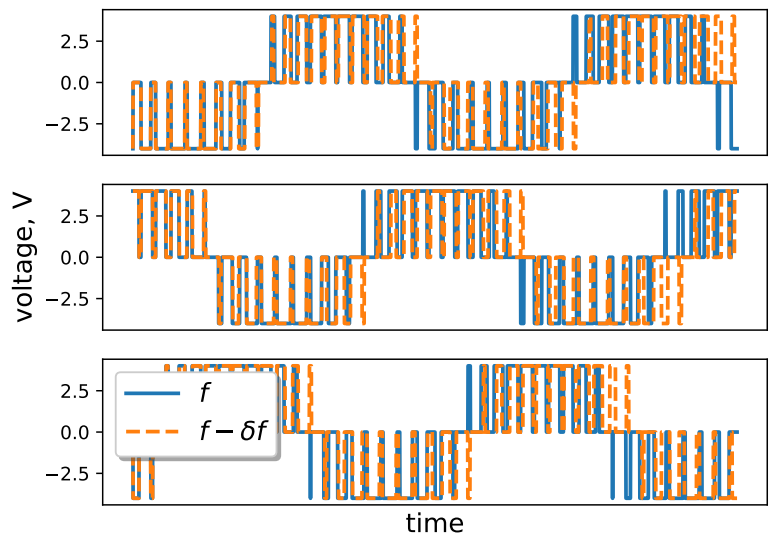

(b)

Figure 5. Example of PWM (5a) and ESC (5b) output signals in case of healthy (blue), and degraded (dashed orange) MOSFET. Figure (5a) shows the $F_{1}$ signal, while (5b) shows all three phase voltages computed from output voltage of a single cell $V \approx 4 \mathrm{~V}$. In this example, $\delta f=0.05 f$.

uncertainty in the switch matrix is defined through meantime-between-failure or similar quantities. An example of the three-phase voltages from a switch failure is shown in figure 6 , where the element $(2,3)$ of the switch matrix in Eq. (1), originally equal to -1 , has been replaced by 0 .

Last, the voltage $V$ in Eq. (1) is the output voltage of the battery, which is modeled as a random variable, already discussed in Subsection 4.1.

\subsection{Motor}

As discussed in Section 3, motor model parameters could be represented by random variables to encapsulate the model parameter uncertainty within the model. According to the
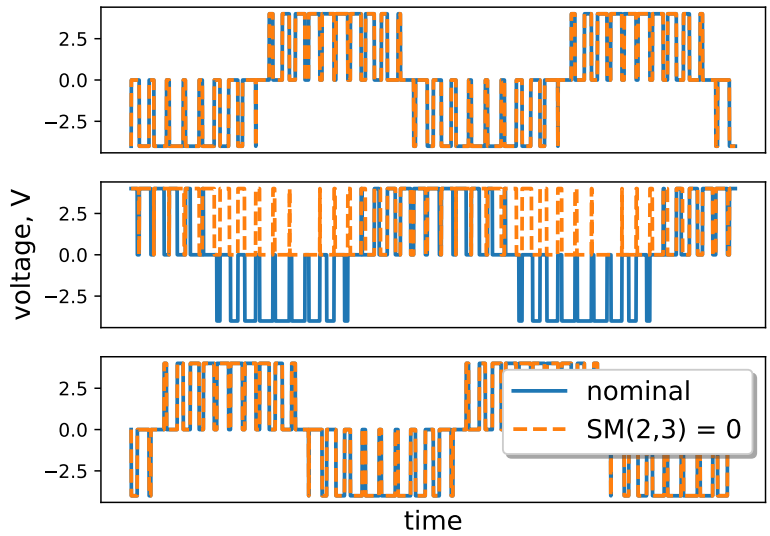

Figure 6. Example of three-phase voltages from ESC with a switch failure. The legend indicates that the switch matrix (SM) element in position $(2,3)$ has been replaced by 0 .

available information, the availability of experimental data, or according to sensitivity analysis performed on the model, the algorithm designer could decide to use a deterministic value for a specific parameter (e.g., electric resistance $R_{s}$ ), and describe the other parameter(s) through probability distributions. In this work the methodology to describe those parameters is presented, and that same methodology could be applied to some (of all) the motor model parameters for statistical assessment of the model. Because of their physical meaning, all model parameters in $\left[B, J, R_{s}, L_{M}, k_{e}\right]^{T}$ have to be strictly positive, $\mathbb{R}^{+}$. As a consequence, Gaussian distributions may not be suitable for describing the uncertainty in those parameters, especially if their value is (relatively) close to 0 . Let us consider, for example, the friction coefficient $B$ and motor inertia $J$. Their distributions can be defined through a log-Normal transformation by simply using:

$$
\begin{aligned}
& \ln B \sim \mathcal{N}\left(\mu_{\ln B}, \sigma_{\ln B}^{2}\right), \\
& \ln J \sim \mathcal{N}\left(\mu_{\ln J}, \sigma_{\ln J}^{2}\right),
\end{aligned}
$$

assuming that $B$ and $J$ are independent random variables (the generalization to the case $\sigma_{\ln B, \ln J} \neq 0$ is straightforward by introducing a multi-variate Normal distribution). Examples of the two log-Normal distributions are shown in figure 7.

An example of angular velocity output from the model in (B.1) using samples from the distributions of $B$ and $J$ is visible in figure 8 . The effect of different inertia values is clearly visible in the transient period necessary to reach the steadystate regime. In order to emphasize the effect of $B$ and $J$ samples, the graph was generated neglecting dynamics effects on the back-emf voltage and external load torque $T_{l}$. 

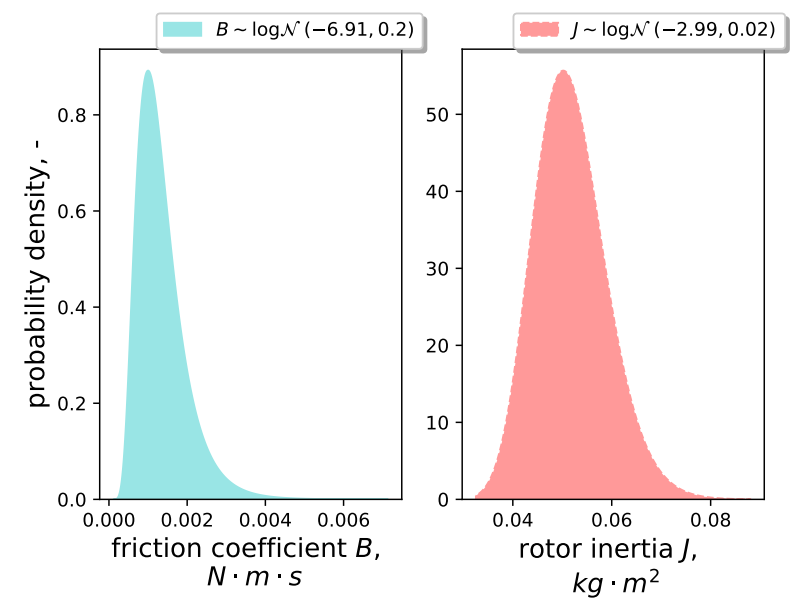

Figure 7. Example of log-Normal probability distributions of $B$ and $J$.

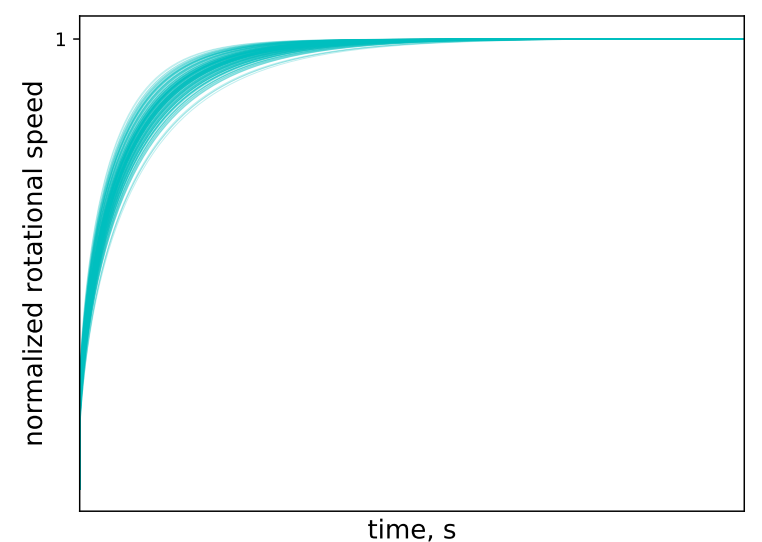

Figure 8. Example of motor speed variations from 0 up to a steady-state regime (nominal speed $\omega_{n}=675 \mathrm{RPM}$ ).

\section{Conclusions}

This work proposed a framework for uncertainty quantification of engineering systems, with a focus towards unmanned aerial vehicle systems and operations. A formal approach starting from a high-level description of uncertainty sources has been utilized to design the framework. Those sources are included within a predictive estimation process, where they are divided into spaces. The key elements within the spaces and their input-output relationships are highlighted. The design of a powertrain health monitoring system for small UAVs has been used as case study. The framework helped identifying modeling properties as well as input and parameters. The health monitoring system can leverage the proposed uncertainty quantification methodology to maximize the performance of fault detection and isolation techniques and mini- mize false alarms. For example, one may refine the definition of fault indicators based on observed uncertainty, or compute uncertainty bounds on those same fault indicators based on the uncertainty observed in models, measure, input and computing methods.

Future work includes both (i) the extension and application of the proposed framework to other sub-systems, and (ii) further development of the proposed powertrain health monitoring system. On the former, uncertainty of manipulated variables controlled using a feedback loop is of great interest for autonomous operations. Sensitivity analysis was not discussed in this work, but is one of the natural, subsequent steps to address uncertainty quantification of the monitored system. The proposed powertrain health monitoring system could be enhanced by studying the effect of input uncertainties into the motor performance and related subsystems. Such a study may help understanding the advantages as well as the limitations of the diagnostic capabilities of the health monitoring system.

\section{ACKNOWLEDGMENT}

This work was supported by the System-Wide Safety (SWS) project under the Airspace Operations and Safety Program within the NASA Aeronautics Research Mission Directorate (ARMD).

\section{REFERENCES}

Albaker, B., \& Rahim, N. (2009). A survey of collision avoidance approaches for unmanned aerial vehicles. In 2009 international conference for technical postgraduates (techpos) (pp. 1-7).

Arulampalam, M. S., Maskell, S., Gordon, N., \& Clapp, T. (2002). A tutorial on particle filters for online nonlinear/non-gaussian bayesian tracking. Signal Processing, IEEE Transactions on, 50(2), 174-188.

Balaban, E., Roychoudhury, I., Spirkovska, L., Sankararaman, S., Kulkarni, C. S., \& Arnon, T. (2017). Dynamic routing of aircraft in the presence of adverse weather using a pomdp framework. In 17th aiaa aviation technology, integration, and operations conference (p. 3429).

Celaya, J. R., Saxena, A., Kulkarni, C. S., Saha, S., \& Goebel, K. (2012, Jan). Prognostics approach for power mosfet under thermal-stress aging. In Proceedings annual reliability and maintainability symposium.

Daigle, M., \& Kulkarni, C. (2013, October). Electrochemistry-based battery modeling for prognostics. In Annual conference of the prognostics and health management society 2013 (p. 249-261).

Drozeski, G. R., Saha, B., \& Vachtsevanos, G. J. (2005). A fault detection and reconfigurable control architecture for unmanned aerial vehicles. In 2005 ieee aerospace conference (pp. 1-9). 
FAA. (2018). Unmanned aerial system (uas) traffic management (utm), concept of operations (Tech. Rep.). Federal Aviation Administration.

Freeman, P., \& Balas, G. J. (2014). Actuation failure modes and effects analysis for a small uav. In American control conference (acc), 2014 (pp. 1292-1297).

Ginart, A., Brown, D., Kalgren, P., \& Roemer, M. (2009). Online ringing characterization as a diagnostic technique for igbts in power drives igbts in power drives. In Ieee transactions on instrumentation and measurement (Vol. 58).

Glasheen, K., Pinto, J., Steiner, M., \& Frew, E. W. (2019). Experimental assessment of local weather forecasts for small unmanned aircraft flight. In Aiaa scitech 2019 forum (p. 1193).

Goebel, K. (2017). Prognostics, the science of making prediction. CreateSpace Independent Publishing Platform (1).

Gorospe, G. E. J., Kulkarni, C. S., Hogge, E., Hsu, A., \& Ownby, N. (2017). A study of the degradation of electronic speed controllers for brushless dc motors. In Asia pacific conference of the prognostics and health management society 2017.

Hoffmann, G., Huang, H., Waslander, S., \& Tomlin, C. (2007). Quadrotor helicopter flight dynamics and control: Theory and experiment. In Aiaa guidance, navigation and control conference and exhibit (p. 6461).

Holtz, J. (1992). Pulsewidth modulation-a survey. IEEE transactions on Industrial Electronics, 39(5), 410420.

Jing, D., \& Haifeng, W. (2013). System health management for unmanned aerial vehicle: conception, state-of-art, framework and challenge. In 2013 ieee 11th international conference on electronic measurement \& instruments (Vol. 2, pp. 859-863).

Johry, A., \& Kapoor, M. (2016). Unmanned aerial vehicle (uav): Fault tolerant design. International Journal of Engineering Technology Science and Research, 3(6), $1-7$.

Jun, M., \& D'Andrea, R. (2003). Path planning for unmanned aerial vehicles in uncertain and adversarial environments. In Cooperative control: models, applications and algorithms (pp. 95-110). Springer.

Karthikeyan, D. K., Sikha, G., \& White, R. E. (2008). Thermodynamic model development for lithium intercalation electrodes. Journal of Power Sources, 185(2), 1398-1407.

King, D. W., Bertapelle, A., \& Moses, C. (2005). Uav failure rate criteria for equivalent level of safety. In International helicopter safety symposium, montreal (Vol. 9).

Kopardekar, P., Rios, J., Prevot, T., Johnson, M., Jung, J., \& Robinson, J. E. (2016). Unmanned aircraft system traffic management (utm) concept of operations. In Aiaa aviation forum.
Krishnakumar, K. S., Kopardekar, P. H., Ippolito, C. A., Melton, J., Stepanyan, V., Sankararaman, S., \& Nikaido, B. (2017). Safe autonomous flight environment (safe50) for the notional last " $50 \mathrm{ft}$ " of operation of "55 lb" class of uas. In Aiaa information systemsaiaainfotech@aerospace (p. 0445).

Langelaan, J. W., Alley, N., \& Neidhoefer, J. (2011). Wind field estimation for small unmanned aerial vehicles. Journal of Guidance, Control, and Dynamics, 34(4), 1016-1030.

Lawler, G. F. (2010). Stochastic calculus: An introduction with applications. American Mathematical Society.

Liu, Y., \& Goebel, K. (2018). Information fusion for national airspace system prognostics. In Phm society conference (Vol. 10).

Pillay, P., \& Krishnan, R. (1989). Modeling, simulation, and analysis of permanent-magnet motor drives. i. the permanent-magnet synchronous motor drive. IEEE Transactions on industry applications, 25(2), 265273.

Rambabu, S. (2007). Modeling and control of a brushless dc motor. Master of Thesis In Power Control and Drives Technology, National Institute of Technology Rourkela.

Rasmussen, C. E., \& Williams, C. K. (2006). Gaussian processes for machine learning (Vol. 2) (No. 3). MIT Press Cambridge, MA.

Saltelli, A., Tarantola, S., Campolongo, F., \& Ratto, M. (2004). Sensitivity analysis in practice: a guide to assessing scientific models. Chichester, England.

Sankararaman, S. (2015). Significance, interpretation, and quantification of uncertainty in prognostics and remaining useful life prediction. Mechanical Systems and Signal Processing, 52, 228-247.

Sankararaman, S. (2017). Towards a computational framework for autonomous decision-making in unmanned aerial vehicles. In Aiaa information systems-aiaa infotech@aerospace (p. 0446).

Sankararaman, S., \& Mahadevan, S. (2013). Separating the contributions of variability and parameter uncertainty in probability distributions. Reliability Engineering \& System Safety, 112, 187-199.

Smith, R. C. (2013). Uncertainty quantification: theory, implementation, and applications (Vol. 12). Society for Industrial and Applied Mathematics (SIAM).

Walker, M. (2010). Next generation prognostics and health management for unmanned aircraft. In 2010 ieee aerospace conference (pp. 1-14).

\section{BIOGRAPHIES}

Matteo Corbetta is a Research Engineer with SGT Inc. at NASA Ames Research Center, Calif. He is investigating uncertainty quantification methods, model-based and datadriven algorithms for diagnostics and prognostics of engineering systems, particularly unmanned aerial vehicles. Prior 
to joining NASA, he worked as R\&D Condition Monitoring Systems Engineer at Siemens Wind Power, Denmark, and as Post-Doctoral researcher and Teaching Assistant at Politecnico di Milano, Italy. He received Ph.D., MSc., and BSc from Politecnico di Milano, Italy. His research interests include stochastic processes, algorithms for uncertainty quantification, machine learning, and system health management. $\mathrm{He}$ is member of AIAA, IEEE, and member of the Editorial Board of the PHM Society.

Chetan S. Kulkarni received the B.E. (Bachelor of Engineering) degree in Electronics and Electrical Engineering from University of Pune, India in 2002 and the M.S. and Ph.D. degrees in Electrical Engineering from Vanderbilt University, Nashville, TN, in 2009 and 2013, respectively. His current research interests include physics-based modeling, model-based diagnosis and prognosis for complex systems. Dr. Kulkarni is a member of the Prognostics and Health Management (PHM) Society, SM AIAA and the SM IEEE.

\section{APPENDICES}

\section{A. ELECTRO-CHEMISTRY BATTERY MODEL}

The model relies on ordinary differential equations to estimate the QoI. The voltage terms of the battery are expressed as functions of the amount of charge in the electrodes. Each electrode, positive (subscript $p$ ) and negative (subscript $n$ ), is split into two volumes, a surface layer (subscript $s$ ) and a bulk layer (subscript $b$ ). The model equations describe how charge moves through those volumes. The rates of change of the charges $q$, defined by $\dot{q}$, are described using

$$
\begin{aligned}
& \dot{q}_{s, p}=i_{a p p}+\dot{q}_{b s, p}, \\
& \dot{q}_{b, p}=-\dot{q}_{b s, p}+i_{a p p}-i_{a p p}, \\
& \dot{q}_{b, n}=-\dot{q}_{b s, n}+i_{a p p}-i_{a p p}, \\
& \dot{q}_{s, n}=-i_{a p p}+\dot{q}_{b s, n},
\end{aligned},
$$

where $i_{a p p}$ is the applied electric current. The term $\dot{q}_{b, s, i}$ describes diffusion from the bulk to surface layer for electrode $l$ :

$$
\dot{q}_{b, s, l}=\frac{1}{D}\left(c_{b, l}-c_{s, l}\right) \quad, \quad \forall \quad l=\{n, p\} \quad,
$$

where subscript $l$ generally refers to the positive $p$ or negative $n$ electrode, and $D$ is the diffusion constant. The Li-ion concentrations are expressed by $c$ :

$$
c_{j, l}=\frac{q_{j, l}}{v_{j, l}} \quad, \quad \forall \quad l=\{n, p\}, \quad j=\{s, b\}
$$

Note now that the following relations hold:

$$
\begin{aligned}
q_{l} & =q_{s, l}+q_{b, l} \quad, \quad \forall \quad l=\{n, p\} \quad, \\
q^{\max } & =q_{s, p}+q_{b, p}+q_{s, n}+q_{b, n} .
\end{aligned}
$$

The mole fraction, utilized to calculate the different voltages, is computed based on charges $q$ :

$$
\begin{aligned}
& x_{l}=\frac{q_{l}}{q^{\max }} \quad, \forall \quad l=\{n, p\}, \\
& x_{j, l}=\frac{q_{j, i}}{q_{j, i}^{\max }} \quad, \forall \quad l=\{n, p\}, \quad j=\{s, b\}
\end{aligned}
$$

where $q^{\max }=q_{p}+q_{n}$ refers to the total amount of available Li-ions. It follows that $x_{p}+x_{n}=1$. For Li-ion batteries, when fully charged, $x_{p}=0.4$ and $x_{n}=0.6$. When fully discharged, $x_{p}=1$ and $x_{n}=0$ (Karthikeyan, Sikha, \& White, 2008).

The overall battery voltage $V$ consists of several electrochemical potentials. Starting from the positive current collector, the equilibrium potential is $V_{U, p}$. This voltage is then reduced by $V_{s, p}$, due to the solid-phase Ohmic resistance, and $V_{\eta, p}$, the surface overpotential, at the positive collector side. The electrolyte Ohmic resistance then causes another drop $V_{e}$. Similarly to the positive electrode, there is a drop $V_{\eta, n}$ at the negative electrode due to the surface overpotential, and a drop $V_{s, n}$ due to the solid-phase Ohmic resistance. The voltage drops again due to the equilibrium potential at the negative current collector $V_{U, n}$. These voltages are described by the following set of equations:

$$
\begin{aligned}
V_{U, l} & =U_{0}+\frac{R T}{n F} \ln \left(\frac{1-x_{s, l}}{x_{s, l}}\right)+V_{\mathrm{INT}, l}, \\
V_{\mathrm{INT}, l} & =\frac{1}{n F} \sum_{k=0}^{N_{l}} A_{l, k}\left(\left(2 x_{l}-1\right)^{k+1}-\frac{2 x_{l} k\left(1-x_{l}\right)}{\left(2 x_{l}-1\right)^{1-k}}\right), \\
V_{o} & =i_{\text {app }} R_{o}, \\
V_{\eta, l} & =\frac{R T}{F \alpha} \operatorname{arcsinh}\left(\frac{J_{l}}{2 J_{l, 0}}\right),
\end{aligned}
$$

where $U_{0}$ is a reference potential, $R$ is the universal gas constant, $T$ is the electrode temperature (in $\mathrm{K}$ ), $n$ is the number of electrons transferred in the reaction ( $n=1$ for Li-ion), and $F$ is Faraday's constant. $V_{\mathrm{INT}, l}$ is the activity correction term ( 0 in the ideal condition). We use the Redlich-Kister expansion with $N_{p}=12$ and $N_{n}=0$ to compute $V_{\text {INT, } l}$ (see (Daigle \& Kulkarni, 2013)). The current density and the total voltage of the battery are expressed by:

$$
\begin{aligned}
J_{l} & =\frac{i}{S_{l}}, \\
J_{l, 0} & =k_{l}\left(1-x_{s, l}\right)^{\alpha}\left(x_{s, l}\right)^{1-\alpha}, \\
V & =V_{U, p}-V_{U, n}-V_{o}^{\prime}-V_{\eta, p}^{\prime}-V_{\eta, n}^{\prime},
\end{aligned}
$$

where $J_{l}$ is the current density, $J_{l, 0}$ is the exchange current density, and $k_{l}$ is a lumped parameter of several constants including a rate coefficient, electrolyte concentration, and maximum ion concentration. The terms to compute the total voltage $V$ are defined by: 


$$
\begin{gathered}
\dot{V}_{o}^{\prime}=\frac{V_{o}-V_{o}^{\prime}}{\tau_{o}}, \\
\dot{V}_{\eta, p}^{\prime}=\frac{V_{\eta, p}-V_{\eta, p}^{\prime}}{\tau_{\eta, p}}, \\
\dot{V}_{\eta, n}^{\prime}=\frac{V_{\eta, n}-V_{\eta, n}^{\prime}}{\tau_{\eta, n}},
\end{gathered}
$$

where the parameters $\tau$ are empirical inertia constants (used since the voltages do not change instantaneously). The state vector, input vector, and output vector of the model are defined follows:

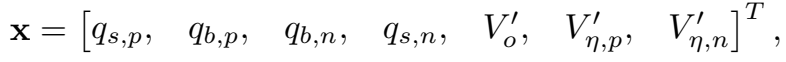

$$
\begin{aligned}
& \mathbf{u}=u=i_{a p p}, \\
& \mathbf{y}=y=V \text {. }
\end{aligned}
$$

Parameter values for a typical Li-ion cell are given in (Daigle \& Kulkarni, 2013).

\section{B. MOTOR DYNAMIC MODEL}

The model describes the dynamics of a three-phase brushless DC motor, with wye-connected stator windings and a permanent magnet as the rotor. Such a model was already described and utilized in (Gorospe et al., 2017). The Newton's second law of motion describes the basic motor dynamics:

$$
\dot{\omega}_{m}=\frac{1}{J}\left(-B \omega_{m}+\left(T_{e}(e, i)-T_{l}\right)\right)
$$

where $\omega_{m}$ is the rotational speed of the rotor, $J$ is the mechanical inertia, $B$ is the friction coefficient, and $T_{l} \propto \omega_{m}$ is the load torque on the rotor. The electrical torque is defined as:

$$
T_{e}(\boldsymbol{e}, \boldsymbol{i})=\frac{1}{\omega_{m}} \boldsymbol{e}^{T} \boldsymbol{i}
$$

where the column-vector $e$ contains the back-emf voltage, $e_{a}, e_{b}$, and $e_{c}$, and $\boldsymbol{i}=\left[i_{a}, i_{b}, i_{c}\right]^{T}$. The back-emf voltages are modeled as trapezoidal waves that are function of the rotor position (Rambabu, 2007; Gorospe et al., 2017), but they are not reported here for the sake of brevity. The interested reader is referred to (Rambabu, 2007) for the trapezoidal wave equations utilized to represent the back-emf voltages. Using the simplifying hypotheses in (Gorospe et al., 2017), we can write the electrical dynamic equations as:

$$
\begin{aligned}
\frac{d}{d t}\left[\begin{array}{l}
i_{a} \\
i_{b}
\end{array}\right] & =-\frac{R_{s}}{L_{M}}\left[\begin{array}{l}
i_{a} \\
i_{b}
\end{array}\right]+\frac{1}{L_{M}}\left[\begin{array}{ll}
2 & 1 \\
1 & 1
\end{array}\right]\left[\begin{array}{l}
v_{a b} \\
v_{b c}
\end{array}\right]+ \\
& -\frac{1}{L_{M}}\left[\begin{array}{ccc}
2 & -1 & -1 \\
1 & 0 & -1
\end{array}\right]\left[\begin{array}{l}
e_{a} \\
e_{b} \\
e_{c}
\end{array}\right],
\end{aligned}
$$

where $L_{M}$ is the difference between self and mutual inductances (assumed equal for each phase), and $R_{s}$ is the resistance of each phase (also assumed identical for the three phases). Equation (B.3) is two-dimensional given the linear dependency of currents and voltages on the three phases, i.e., $i_{a}+i_{b}+i_{c}=0$. Further information are not reported for the sake of brevity. The interested reader is referred to (Gorospe et al., 2017) for more details about the motor dynamic model. 